\title{
El diario Le Monde y la intervención francesa en el Sahara Occidental
}

\author{
Guadalupe Pérez García \\ Doctora Universidad Complutense de Madrid
}

\section{Resumen}

En la actualidad, Francia juega un papel primordial en la evolución de las relaciones hispanomarroquíes. Durante el franquismo su intervención fue determinante en el último período colonial de España en el Sahara Occidental, así como en su "descolonización" o, si se prefiere, en su abandono en 1975. El diario Le Monde, cuya información internacional gozaba ya entonces de un enorme prestigio ganado a pulso con su crítica cobertura contra la guerra de Indochina y en la independencia de Argelia, cubrió este periodo de una manera particular.

\begin{abstract}
Nowadays, France plays a fundamental role in the evolution of the relations between Spain and Morocco. In the 1970s its intervention was essential in the last colonial period of Spain in the Western Sahara, as well as in its withdrawal in 1975. The French newspaper Le Monde had then an enormous prestige thanks to its impartial position in relation to Indochina war and Algerian independence, so its role in Spanish withdrawal was also very important.
\end{abstract}

Palabras clave: Le Monde, Comunicación, Sahara Occidental, Propaganda Key words: Le Monde, Communications, Western Sahara, Propaganda

\section{LAS RELACIONES hISPANO-FRANCESAS EN EL NORTE DE ÁFRICA}

a en 1912, como es sabido, España y Francia dividen Marruecos en dos protectorados; desde entonces las relaciones entre ambas naciones oscilarán entre la amistad (gracias a Francia vence España a Abd El Krim) y la beligerancia. Durante la segunda guerra mundial España presentará a Berlín un memorándum en que solicitaba la cesión del Marruecos francés, el Oranesado y la ampliación del Sahara Occidental y territorios españoles en el Golfo de Guinea. Con el fin de la II Guerra Mundial se produjo una mejora de relaciones, lo que lleva a una reunión del Alto Comisario español, Teniente General José Enrique Varela con el residente general francés, el General Alphonse Juin, en 1948. En ella se acordó oponerse conjuntamente a las peticiones nacionalistas en África, si bien en 1951 las relaciones empeoraron nuevamente. 
Francia llegó a trasladar al temido Abd El Krim al mediodía de Francia para facilitar la organización de un foco de perturbaciones próximo a la zona española. España proporcionará un considerable apoyo al nacionalismo marroquí antifrancés y se pronunció a favor del monarca marroquí cuando fue deportado. El revanchismo franquista llevará, junto a la propia evolución de la zona francesa, a la pérdida irreversible del protectorado español. Sin embargo, será en los territorios del África Occidental donde inicien tímidamente una mayor cordialidad.

Desde septiembre de 1957 los Estados Mayores de Francia y España comenzarán las conversaciones sobre una colaboración militar frente a una posible insurrección en el Sahara, Ifni o Mauritania, alentada por el nacionalismo marroquí del partido nacionalista Istiqlal. El ataque del Ejército de Liberación, orquestado desde Rabat, se cierne en primer lugar sobre la zona de Ifni el 23 de noviembre y se extiende con mayor virulencia al Sahara a partir del mes de diciembre y enero de 1958. Ifni había sido excluido de la colaboración franco-española por encontrarse demasiado alejado de los intereses franceses en Mauritania; sin embargo, en el Sahara la colaboración será completa. Así nacía la Operación Ouragan, dividida en Ecouvillon y Teide. Tras la entrega por parte de España del territorio de Tarfaya a Rabat, la paz quedará restablecida en el territorio a partir de junio de 1958.

Sin embargo, la colaboración militar había sido convenientemente silenciada tanto en Francia como en España: la comparecencia del ministro del Ejército Antonio Barroso sobre el asunto ante las Cortes el 21 de diciembre no fue difundida, al igual que la decisión del Consejo de Ministros francés el 30 de diciembre de cooperación militar con España. Éste último aceptará la operación militar conjunta movido por varias razones: en primer lugar, resultaba obvia la amenaza que para Mauritania suponía el Ejército de Liberación; en segundo lugar, decía responder a las peticiones de la población mauritana y, en tercero y último, necesitaba la seguridad financiera para la compañía Miferma, que actuaba en la zona. Sin embargo, su complicada posición internacional (colaboración con Franco, lucha colonial en Argelia y relaciones con Marruecos) hacen que París trate de camuflar la nueva situación.

España mantendrá una presencia precaria en el territorio de Ifni, que finalmente será retrocedido a Marruecos once años después, en 1969. Comenzaba entonces el último lustro de presencia española en el Sahara Occidental, período complicado que culminará en el abandono del territorio por el régimen franquista en febrero de 1976, según lo establecido en el Acuerdo de Madrid cuatro meses antes. Francia por su parte vivía su larga agonía en Argelia y concedía la independencia a Mauritania, territorio colindante con el Sahara Español.

En tal contexto, se produjo la intervención, plenamente promarroquí, de Valéry Giscard D'Estaing en la Marcha Verde.

En julio de 1975, Henry Kissinger recibió un informe de la CIA en el que se relataba una preocupante inestabilidad interna de Marruecos, así como una complicada situación exterior marcada por las difíciles relaciones con Argelia. Como 
consecuencia, Estados Unidos y Francia decidirán apoyar al aliado conservador frente al socialismo argelino y tomarán posición a favor de Marruecos en el asunto de la reivindicación del Sahara. De hecho, puede decirse que parte de la organización logística de la Marcha, así como la intervención mauritana en el conflicto fueron orquestadas en el Quai D'Orsai.

Valéry Giscard, recién elegido presidente de Francia, era a su vez amigo de Hassan II, al tiempo que los intereses económicos franceses en Marruecos desde la época del protectorado continuaban siendo primordiales. Se añadía además una razón de Estado: con un Sahara marroquí todo el África Occidental sería francófona. Como resultado, D’Estaing movilizó todos los recursos para convencer al presidente mauritano de que favoreciera los intereses del monarca alauí. El presidente de Túnez, Habib Burguiba y el de Senegal, Léopold Senghor, le ayudaron en la tarea y poco a poco Mauritania comenzó a aproximarse a Rabat.

Tras el acuerdo de Madrid, la colaboración francesa con los marroquíes resultaba evidente, lo que provocará que el Polisario convierta a sus nacionales en objetivo de sus secuestros. El 25 de diciembre de 1975 dos cooperantes franceses desaparecían en la región de Tarfaya; poco después, cinco jóvenes eran atacados al norte del Sahara. A primeros de enero, dos técnicos franceses y cuatro obreros marroquíes eran hechos prisioneros cerca de Bu Craa.

Por su parte, el primer ministro marroquí, Ahmed Osman, alababa en visita a París la posición francesa, al mismo tiempo que le encargaba setenta y cinco aviones Mirage F-1, lo que acrecentó la tensión franco-argelina. Como respuesta, el ex primer ministro Messmer y el presidente Giscard D'Estaing tomaron claro partido a favor de Marruecos, de forma que negaron la existencia de un pueblo saharaui. Pese a ello, Francia ofrecerá su colaboración en el conflicto, propuesta que no sería aceptada ni por Argelia ni por el Frente Polisario. Por el contrario, el partido socialista francés criticaba la actitud del gobierno y Gaston Defferre se lamentaba ante el embajador argelino del papel jugado por Francia en el conflicto saharaui. Miterrand declaraba el 25 de febrero en Argel ante una delegación del Frente Polisario su convencimiento sobre el derecho de autodeterminación saharaui.

Sin embargo, Francia había logrado su propósito de mantener la estabilidad marroquí, así como sus intereses económicos, que en 1974 superaban los 881 millones de francos en contratos y en 1975 se acercaban a los 742 millones. París construía así un eje junto a Madrid-Rabat-Nuakchott-Dakar que se enfrentaba a Argel.

\section{COBERTURA DE LE MONDE EN LA GUERRA DE IFNI-SAHARA (1957-1958). LA OMISIÓN DE LA COLABORACIÓN FRANCO-ESPAÑOLA}

Para analizar la cobertura que Le Monde realizó sobre la última guerra colonial en la que se embarcó España, la llamada "guerrita" de Ifni a finales de los 
cincuenta, resultaron pertinentes al objetivo de este estudio un total de 56 artículos (13 de los cuales fueron incluso portada) en un período comprendido entre el 23 de noviembre de 1957 y el 30 de junio de $1958^{1}$. De ello se deduce que Francia dedicó una notable atención a los hechos, seguramente inducida por el efecto de arrastre que en aquellos momentos ejercía el caso argelino sobre cualquier conflicto en el norte de África.

Será el 26 de noviembre -incluso un día antes a la fecha elegida por el gobierno español para dar su primera nota oficial- cuando Le Monde cubra el ataque por sorpresa del Ejército de Liberación en Ifni ${ }^{2}$. A su vez, con una óptica más nacional, se preocupa por la incidencia que este ataque podría tener sobre las proposiciones tunecino-marroquíes sobre el asunto argelino ${ }^{3}$. De hecho, a partir de ese mismo día, comienza a incluir las informaciones referentes a la guerra de Ifni y Sahara dentro de una misma sección dedicada al norte de África: "Les événements d'Afrique du Nord". Su cobertura estará asegurada por dos corresponsales: Jean Lefèvre, que se desplazará por Marruecos en función de la evolución del conflicto y Louis Gravier, siempre desde Rabat.

Paradójicamente, el diario francés se convertía en una frecuente referencia para la prensa española, la cual a priori, debía tener un mejor acceso a las fuentes. Así por ejemplo, la posibilidad de entregar Tarfaya la anticipaba $A B C$ citando a $L e$ Monde:

"El periódico francés subraya que Madrid estaría dispuesto a estudiar la transferencia de la zona sur del Protectorado español en Marruecos, ahora que las fuerzas armadas reales controlan efectivamente las regiones meridionales de Marruecos y por haberse desintegrado las bandas del titulado 'Ejército de Liberación', como consecuencia de los reveses militares que han sufrido en el Sahara" ${ }^{4}$

\footnotetext{
${ }^{1}$ La última noticia que incluimos en este período está datada el 19 de abril de 1958 (LEFÈVRE, Jean: "L'armée marocaine constate qu'elle ne pourrait subsister faute d'accord avec les Espagnols", Le Monde, 19 de abril de 1958, p. 3), ya que desde esta fecha hasta al 30 de junio Le Monde no dedicó ningún artículo al conflicto.

2 "Nouveaux incidents à Ifni", Le Monde, 26 de noviembre de 1957, p. 1. Aunque el adjetivo "nouveaux" parece indicar que ya se había informado sobre el ataque con anterioridad, lo cierto es que ésta es la primera referencia a los hechos del 23 de noviembre. En todo caso, el titular se refiere a los incidentes sucedidos antes de esta fecha.

${ }^{3}$ Esta propuesta había sido recogida días antes, en el artículo "Le communiqué officiel sur les entretiens de Rabat", Le Monde, 23 de noviembre de 1957, p. 2

4 "Las relaciones hispano-marroquíes", $A B C, 28$ de marzo de 1958, p. 39
} 
También se utilizarán sus informaciones para resaltar la figura del Caudillo:

\begin{abstract}
"El diario Le Monde, al ocuparse del discurso de fin de año del Generalísimo Franco, del que da una amplia reseña, subraya de un modo especial la moderación con que se ha expresado respecto a Ifni, afirmando que la alocución es totalmente reveladora de la mentalidad del Jefe de Estado español y añade que, indudablemente, el antiguo Jefe de la Legión española se acuerda aún con cierta emoción de sus campañas africanas y de los marroquíes del antiguo Protectorado, con quienes gustaba parlamentar"
\end{abstract}

La estructura de las noticias de Le Monde durante estos primeros momentos de la guerra será siempre la misma: tras reiterar las dificultades para informar desde el escenario de los hechos, Le Monde hará continua referencia a la guerra de propaganda y contrapropaganda entre Marruecos y España, para finalmente indicar el papel de Francia en los acontecimientos. Así por ejemplo, en una crónica de Jean Lefèvre ${ }^{6}$, el periodista se queja de las informaciones fragmentarias ofrecidas por el gobierno español y marroquí y opone las versiones diferentes ofrecidas por el Ejército español (que acusa al Ejército de Liberación de los ataques) y la de diario del Istiqlal Al Alam, que habla más bien de una insurrección interna de los ifneños en la que Rabat nada tendría que ver. Finalmente niega, citando fuentes próximas al Estado Mayor francés en Rabat, cualquier tipo de intervención francesa en Ifni.

La cobertura de Le Monde resulta interesante en la medida en que también desenmascara la guerra de propaganda entre los medios españoles y marroquíes. No sólo en lo que respecta a quién corresponden las victorias y derrotas militares (convenientemente exaltadas y silenciadas en Rabat y Madrid) ${ }^{7}$, sino también en determinadas estrategias, como la inventada por algunos voceros del franquismo sobre la posible intervención de una "mano negra comunista" en el norte de África, que por otra parte, nunca fue probada ${ }^{8}$.

Finalmente, Le Monde contribuyó a la minimización ante la opinión pública de la participación francesa junto a la España franquista en el Sahara, tal y como deseaba París. Entre corchetes y en un tamaño de letra inferior al del resto de la información, encontramos incluso un desmentido el 17 de diciembre de $1957^{\circ}$.

\footnotetext{
5 "Le Monde elogia la ponderación del Caudillo en Ifni", Arriba, 9 de enero de 1958, p. 10

${ }^{6}$ LEFÈVRE, Jean: "Des centaines d'irréguliers de l'Armée de libération marocaine aux prises avec les garnisons renforcées des postes frontières", Le Monde, 26 de noviembre de 1957, p. 3

7 "Situation confuse dans l'enclave d'Ifni", Le Monde, 10 de diciembre de 1957, p. 1

${ }^{8}$ L.G. "M. Bekkai: le prince Hassan a évité le pire à Ifni", Le Monde, 20 de diciembre de 1957, p. 6

9 "Incertitude pour Ifni: compromis ou opération d'envergure?", Le Monde, 17 de diciembre de 1957 , p. 5
} 
Al día siguiente, confirma el distanciamiento francés ante un conflicto entre dos países amigos ${ }^{10}$ y por fin en enero el periódico insinúa la trama de relaciones internacionales que podría empujar a Francia a actuar, pero no se decanta por ninguna de las posibilidades y no aclara que en esos momentos la cooperación militar franco-española sea ya un hecho

Posteriormente, Le Monde continuará haciéndose eco de los desmentidos oficiales, para terminar admitiendo, unos días después, la intervención francesa al sur del paralelo $27^{\circ}-40^{, 12}$.

\section{LA PROGRESIVA CONSTRUCCIÓN DE LAS BUENAS RELACIONES FRANCO-MARROQUÍES}

La etapa posterior a la guerra fue un período de transición en el Sahara e Ifni y la atención de la prensa francesa fue obviamente menor, si bien ésta se concentró en dos acontecimientos: la sucesión del monarca alauí y el secuestro de un grupo internacional de técnicos petrolíferos que trabajaban en el Sahara por cuenta de España en 1961.

A través del relato que Le Monde construye en torno a la muerte y funerales de Mohamed V, se puede apreciar un trato muy favorable del periódico hacia la monarquía marroquí. Se halaga al rey profusamente y a partir de la sensación de unanimidad y duelo general que narra el rotativo, se deduce la legitimidad de su institución. Así puede apreciarse en tres noticias: "Après la mort de Mohamed V Hassan II ne voudra pas être un roi imposé, mais accepté, déclare la ministre marocain de l'information" "13 "Journée de deuil au Maroc: Jamais Mohamed V n'avait été mieux qu'au delà de la mort le 'sultan des Carrières centrales" "14 y "Le roi Hassan II a été solennellement intronisé,"15.

El siguiente hecho sobre el que se centró la atención francesa fue el secuestro de los técnicos petrolíferos, entre los que se encontraban súbditos franceses y con toda seguridad orquestado por Rabat, en marzo de 1961. Sin embargo, la cobertura que Le Monde concedió a este episodio fue muy similar a la que realizó durante

\footnotetext{
10 "Mohamed V se saisit du dossier hispano-marocain", Le Monde, 18 de diciembre de 1957, p. 7

"L'insuccès de la négociation sur le retait de la peseta aggrave les difficultés hispanomarocaines que Paris considère avec attention", Le Monde, 11 de enero de 158, p. 1

12 "L'ambassade de France à Rabat dément une collaboration militaire franco-espagnole dénoncée par M. Balafrej", Le Monde, 21 de febrero de 1958, p. 2

13 "Après la mort de Mohamed V Hassan II ne voudra pas être un roi imposé, mais accepté, déclare la ministre marocain de l'information", Le Monde, 1 de marzo de 1961, p. 3

${ }^{14}$ GRAVIER, Louis: "Journée de deuil au Maroc: Jamais Mohamed V n'avait été mieux qu'au delà de la mort le 'sultan des Carrières centrales", Le Monde, 2 de marzo de 1961, p. 3

${ }^{15}$ GRAVIER, Louis: "Le roi Hassan II a été solennellement intronisé", Le Monde, 4 de marzo de 1961 , p. 1
} 
la guerra: pondrá de relieve la guerra de propaganda entre Marruecos y España para decantarse por la versión más probable.

En la primera noticia sobre el hecho, Le Monde considera que se trata de un secuestro perpetrado por elementos marroquíes ${ }^{16}$. Sin embargo, se hace eco de las dos versiones contrapuestas: la marroquí, que acusa a los técnicos de haber franqueado la frontera del Sahara, razón por la cual éstos habrían sido detenidos usurpando su territorio, y la española, por la que el Ejército de Liberación los habría secuestrado en la zona del Sahara Español ${ }^{17}$.

Finalmente, el papel que le concede a su propio país en los acontecimientos es inexistente. En primer lugar, ha de tenerse en cuenta la consolidación de unas buenas relaciones franco-marroquíes, como puede observarse en el trato favorable que dispensó el periódico a la monarquía alauí durante los funerales de Mohamed V y su sucesión por Hassan II. En segundo lugar, se ha de tener en cuenta que uno de los técnicos petrolíferos secuestrados en marzo de 1961 era francés y que también lo era uno de los embajadores a los que el rey alauí en persona ofreció un discurso sobre la legitimidad del "Gran Marruecos" durante la ceremonia de liberación de los técnicos. Sin embargo, Le Monde no implica a Francia de ningún modo, ni siquiera con una declaración oficial de su gobierno y reduce los hechos a un enfrentamiento entre España y Marruecos. $Y$ es que se trataba éste de un período en el que Rabat ofrecía a Francia la explotación conjunta del Sahara a través de compañías mixtas ${ }^{18}$.

\section{LA RETROCESIÓN DE IFNI Y EL ABANDONO DEL SAHARA}

Durante esta etapa, Le Monde dedicará al último período colonial de España en Ifni y Sahara un total de 128 artículos, de las cuales veinticuatro ocuparon la portada del diario. Si se podría concluir que la atención de Le Monde durante este período se incrementa de nuevo tras una etapa de cierto olvido, esta fase engloba dos hechos diferentes: la retrocesión de Ifni en 1969, a la que dedica sólo tres noticias, y el período en el que se gesta el abandono del Sahara (julio de 1974febrero de 1976), sobre el que versan las 125 restantes.

Ifni se trataba de una zona en la que Francia se había negado a participar junto a las fuerzas españolas para expulsar al Ejército de Liberación en la guerra de finales de los cincuenta. En junio de 1969 fue retrocedido a Marruecos de común acuerdo, acontecimiento que poco interesó a los franceses. Le Monde sólo le

\footnotetext{
16 "Les chercheurs de pétrole enlevés au Sahara par del éléments marocains seraient immédiatement ramenés en Espagne", Le Monde, 15 de marzo de 1957, p. 2

${ }^{17}$ Ibídem

${ }^{18}$ GRAVIER, Louis: "M. Alaoui èvoque la thèse marocaine sur l'explotation économique du Sahara", Le Monde, 24 de marzo de 1961, p. 4
} 
dedicó tres noticias ${ }^{19}$, de las que dos hacían mención a la retrocesión sólo con motivo del viaje de Hassan II a Madrid.

Con respecto al proceso que llevó a la Marcha Verde y al Acuerdo de Madrid sobre el Sahara, la posición que España pretendió reflejar internacionalmente fue la de un país respetuoso con las decisiones de la ONU y, ante todo, preocupado por el respeto al derecho de autodeterminación de los saharauis. Le Monde recoge, por ejemplo, un documento de la Dirección General del Sahara de septiembre de 1974 en el que resume esta posición del régimen franquista ${ }^{20}$. Unos meses después, sin embargo, constata la contradicción y el giro que España realiza con respecto a las resoluciones de la ONU, al publicar un comunicado tras un consejo de ministros en el que declara estar dispuesta a abandonar el territorio sin más ${ }^{21}$. Algunos sectores, representados en la figura de Jaime de Piniés en las Naciones Unidas, reivindicarán el derecho a la autodeterminación del pueblo saharaui aún durante la Marcha Verde ${ }^{22}$. Pero finalmente Le Monde pondrá de manifiesto la contradicción de los españoles tras la firma del Acuerdo de Madrid ${ }^{23}$ y la existencia de dos "lobbies" contrapuestos en el gobierno franquista con respecto al tema del Sahara, uno pro-marroquí y otro pro-argelino ${ }^{24}$.

Marruecos, al igual que España, buscará legitimar sus intereses con la defensa del pueblo saharaui, al que considera naturalmente ligado al Gran Imperio Cherifiano, de forma que habla de "libération du territoire" ". Hassan II se afanará igualmente en resaltar los lazos comunes al norte y al sur del paralelo $27^{\circ} 40_{27}^{, 26}$ y criticará a España por su intención de crear en el Sahara un estado fantoche ${ }^{27}$.

En cuanto al Frente Polisario, se recogen sus acciones contra la presencia española en el territorio y sus manifestaciones ante la comisión de la ONU, extremo que fue silenciado en los medios españoles ${ }^{28}$. Posteriormente, ante el

19 "Le Roi Hassan II se rend en Espagne", Le Monde, 28 de junio de 1969, p. 9; "Le roi Hassan II du Maroc séjourne en visite privée à Madrid", Le Monde, 1 de julio de 1969, p. 4 y "Remise du territoire d'Ifni aux autorités chérifiennes", Le Monde, 2 de julio de 1969, p. 6

20 "Madrid: les sahariens sont nos seuls interlocuteurs", Le Monde, 10 de septiembre de 1974, p. 2

21 “Dégagement espagnol au Sahara?", Le Monde, 26 de mayo de 1975, p. 1

22 "Le Conseil de Sécurité se réunit à la demande de l'Espagne", Le Monde, 21 de octubre de 1975, p. 4

${ }^{23}$ NIEDERGANG, Marcel: "Le Maroc, l'Espagne et la Mauritanie seraient parvenus à un accord”, Le Monde, 15 de noviembre de 1975, p. 2

${ }^{24}$ M. N. "Madrid: No pasarán", Le Monde, 7 de noviembre de 1975, p. 4

${ }^{25}$ DECRAENE, Philippe: "Nouakchott: éviter la rupture avec le Maroc", Le Monde, 17 de septiembre de 1974 , p. 6

${ }^{26}$ GRAVIER, Louis: "Hassan II a rappelé à ses sujets sahraouis les liens d'allégeance les unissant au sultanat", Le Monde, 25 de octubre de 1975, p. 8

${ }^{27}$ GRAVIER, Louis: "Nous n'admettrons pas qu'un État fantoche soit créé au Sahara espagnol, déclare le roi Hassan II", Le Monde, 10 de julio de 1974, p. 2

28 "Tandis que les incidents se multiplient dans le territoire, la bataille juridique à propos du Sahara espagnol est entrée dans une nouvelle phase", Le Monde, 15 de mayo de 1975, p. 6 
comienzo de la Marcha Verde, Le Monde anunció que el Polisario preparaba una contramarcha frente a Marruecos, información que tuvo que ser desmentida días después ${ }^{29}$.

Finalmente, resta analizar cuál fue la cobertura del diario sobre la solapada intervención francesa a favor de Rabat. Ésta comenzó con el viaje que el presidente francés, Valéry Giscard D’Estaing realizó a Marruecos en mayo de 1975. En él se ponen de manifiesto las buenas relaciones entre ambos países y Hassan II agradece el voto francés favorable a la resolución presentada por Marruecos en la Asamblea General de Naciones Unidas, cuyo objetivo era someter el asunto del

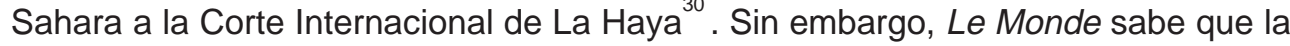
prensa marroquí no es ajena a la discreción francesa y recuerda que el diario L'Opinion, órgano del Istiqlal, ha destacado que el presidente francés no hizo referencia en su discurso a las reivindicaciones de Rabat sobre el Sahara Español ${ }^{31}$.

Después, en una fase posterior del conflicto y una vez firmado el acuerdo de Madrid, Le Monde recogerá las críticas de la prensa argelina y del Polisario, con las que acusaban a Francia de colaborar solapadamente con Rabat ${ }^{32}$. El 1 de febrero publicará una entrevista al presidente D’Estaing, en la que éste negaba su participación activa en el conflicto, si bien se manifestaba de un modo abierto a favor de la solución marroquí y en contra de la independencia del Sahara ${ }^{33}$.

Louis Gravier continuó siendo el corresponsal de Le Monde en Rabat durante el período que va de 1957 a 1975. Su información se caracterizará igualmente por la contextualización de los hechos recurriendo siempre a fuentes marroquíes: desde el mismo Ejército de Liberación hasta los medios oficiales o la prensa de Rabat ${ }^{34}$. A sus crónicas se opondrán las de Marcel Niedergang en Madrid, corresponsal que analizará las repercusiones que el cambio de régimen habrá de tener sobre el desarrollo de los acontecimientos en el Sahara. A través de sus artículos podremos conocer información que se silenciaba en España antes de la firma del Acuerdo de Madrid $^{35}$.

\footnotetext{
29 "Le Conseil de Sécurité se réunit à la demande de l'Espagne", cit.

30 "Hassan II: nos deux pays se rencontrent", Le Monde, 6 de mayo de 1975, p. 2

${ }^{31}$ JUNQUA, Daniel y GRAVIER, Louis: "M. Giscard d'Estaing s'est engagé à remédier aux difficultés de la colonie française au Maroc", Le Monde, 7 de mayo de 1975, p. 6

32 "Le rôle de Paris est loin d'être neutre', écrit El Moudjahid", Le Monde, 7 de enero de 1976, p. 3

33 "Les déclarations de M. Giscard D’Estaing", Le Monde, 1-2 de febrero de 1976, p. 3

${ }^{34}$ Véase como ejemplo GRAVIER, Louis: "Au Maroc la presse dénonce l'attitude de l'Algérie à propos du Sahara espagnol", Le Monde, 29 de abril de 1975

${ }^{35}$ NIEDERGANG, Marcel: "Le conflit du Sahara occidental a précipité la venue au pouvoir de Juan Carlos", Le Monde, 1 de noviembre de 1975, pp. 1 y 2.
} 
Finalmente, desde El Aaiun y Tarfaya, en plena Marcha Verde, envió sus crónicas el periodista Jean Michel Durand-Souffland. Se caracterizó, al igual que Jean Lefèvre, por un estilo muy personal, con el que narrará las experiencias de los enviados especiales en la Marcha (relató por ejemplo, las malas condiciones sanitarias en las que les mantuvieron y la dificultad para informar desde la misma, en la que más de 150 periodistas debían contentarse con tan sólo tres teléfonos) ${ }^{36}$. Además, fue más allá de la mera propaganda de Rabat, denunciando la ausencia de las clases acomodadas en la misma ${ }^{37}$.

Con respecto a la sección en la que suelen insertarse las noticias sobre Ifni o Sahara, si bien se ubican siempre en el área de internacional, lo cierto es que la sección ad hoc creada específicamente por Le Monde varía mucho con el tiempo. Durante la guerra de 1957-1958 era frecuente encontrar el epígrafe general de "Les événements d'Afrique du Nord", bajo el que se encontraban, además de las noticias referentes a Ifni y Sahara, los graves acontecimientos de Argelia, que tanto preocupaban a la opinión pública francesa. Tal epígrafe fue el utilizado frecuentemente, si bien en ocasiones se sustituía por otros similares, como "Le conflit nordafricain" o "L'évolution du monde arabe". Otras veces se refería al problema argelino: "Les bons offices et la situation en Algérie", "Les perspectives de négociations et la situation en Algérie" o "La situation en Algérie et les préparatifs de la négociation".

En los años setenta las noticias sobre el Sahara ya no se encuadran en el conflicto de Argelia, sino se incluyen dentro de una subsección más general denominada "Afrique". De hecho, aunque la noticia hiciese referencia a un problema interno español referido al Sahara, aparecía bajo esta sección y no bajo la de "Europe". Con el recrudecimiento del conflicto y el avance de la Marcha Verde, Le Monde añadirá a la sección antetítulos como "Le conflit du Sahara occidental", "L'avenir du Sahara Occidental" que irán evolucionando hacia "La Marche Verte des marocains au Sahara occidental a commencé", "La décolonisation du Sahara Occidental" o "Les conséquences de la crise du Sahara occidental".

Finalmente Le Monde dedicó a diferentes figuras relacionadas con el conflicto un total de cuatro caricaturas. La primera de ellas se publica con motivo de la visita de Giscard d'Estaing a Hassan II en mayo de 1975 y representa a ambos agarrados de la mano sobre una alfombra con la bandera marroqui ${ }^{38}$. El dibujo hace una referencia obvia a las buenas relaciones, incluso personales, entre ambos.

${ }^{36}$ DURAND-SOUFFLAND, J-M. "Les bivouacs dans le désert", Le Monde, 9-10 de noviembre de 1975, p. 2

${ }^{37}$ DURAND-SOUFFLAND, J.M. "Avec les volontaires à Tarfaya des croisés en guenilles", Le Monde, 2-3 de noviembre de 1975, p. 4

38 JUNQUA, Daniel y GRAVIER, Louis: "M. Giscard d'Estaing s'est engagé à remédier aux difficultés de la colonie française au Maroc", cit. 


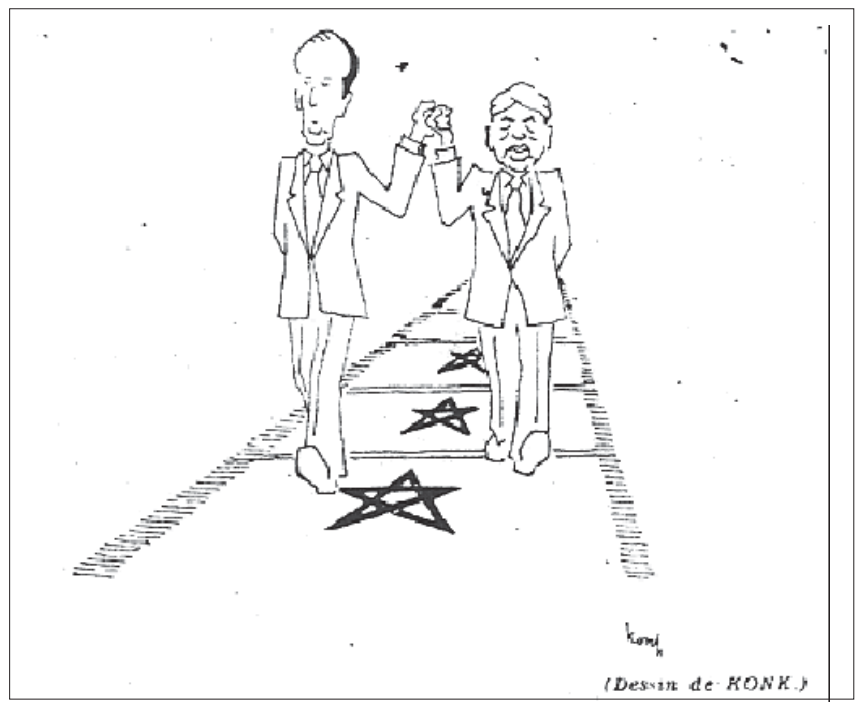

Fig. 1. Viñeta en Le Monde, 7 de mayo de 1975, p. 6

La segunda resulta muy reveladora con respecto a la ambigüedad de la política que España mantendría en el Sahara, pues muestra un Franco muy envejecido en el desierto, vestido con un uniforme que le queda enorme y desenvainando una espada rota ${ }^{39}$.

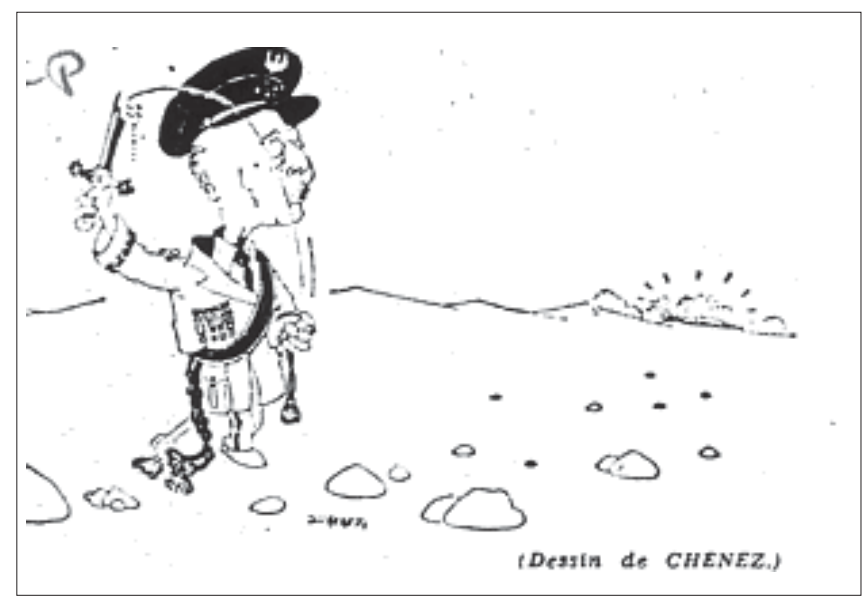

Fig. 2. Viñeta en Le Monde, 22 de octubre de 1975, p. 2

39 “L’Espagne assumera ses responsabilités de puissance administrante, déclare au Conseil de sécurité le représentant de Madrid", Le Monde, 22 de octubre de 1975, p. 2 
La tercera hace referencia a la visita de don Juan Carlos al Sahara a principios de noviembre de 1975 y en ella aparece vestido de uniforme en el desierto frente a varios saharauis sonrientes. De su boca sale un bocadillo que reza "Vive l'autodetermination et la démocratie" ${ }^{40}$.

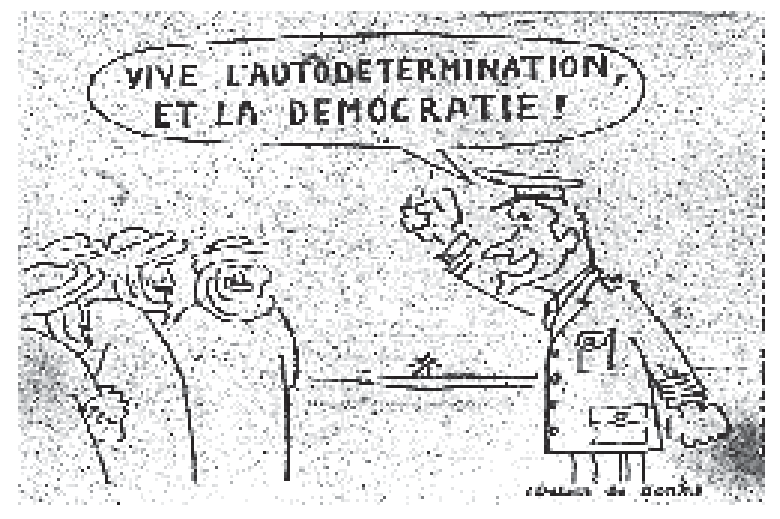

Fig. 3. Viñeta en Le Monde, 4 de noviembre de 1975, p. 1

Por último, la cuarta se cuestiona la homogeneidad de la composición de la Marcha Verde en cuanto a clases sociales e insinúa que ésta es una manipulación populista del monarca, al que representa con una espada en un coche de lujo desde el que dirige a una multitud sonriente pero harapienta ${ }^{41}$.

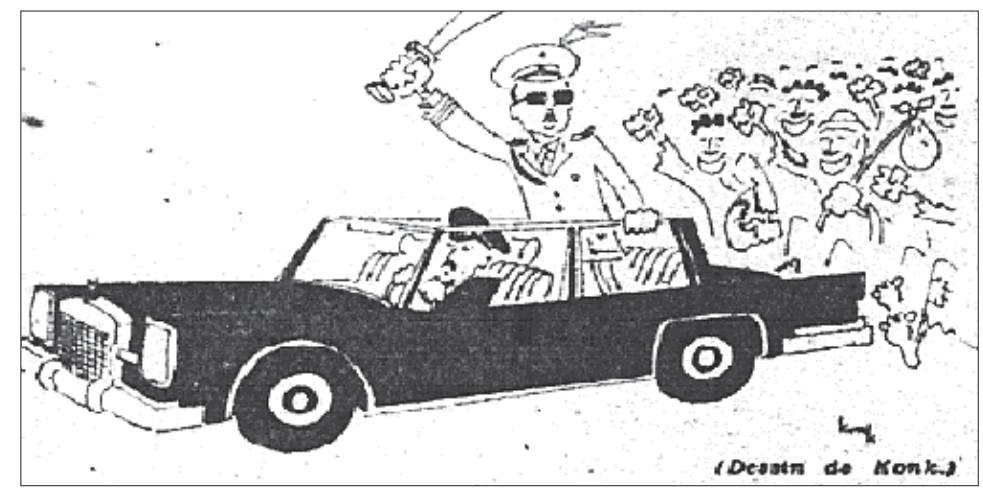

Fig. 4. Viñeta en Le Monde, 7 de noviembre de 1975, p. 4

\footnotetext{
${ }^{40}$ NIEDERGANG, Marcel: "L'Espagne et le Maroc reprennent le dialogue sur le Sahara", Le Monde, 4 de noviembre de 1975, pp. 1 y 4

41 "Tarfaya: la frontière est franchie", Le Monde, 7 de noviembre de 1975, p. 4
} 


\section{CONCLUSIONES}

El papel jugado por Francia en el último período colonial de España en Ifni y Sahara fue notable. En la guerra de los cincuenta colaboró con las tropas franquistas en territorio saharaui para expulsar a un Ejército de Liberación marroquí que también suponía una amenaza para sus intereses en Mauritania. En los años setenta, Valéry Giscard d'Estaing, amigo personal de Hassan II, intervino a favor de Marruecos: apoyó logísticamente la Marcha Verde, le proporcionó armamento frente a Argelia y forzó a Mauritania para que se colocase en el bando marroquí. La estrategia de Le Monde en ambos casos fue, frente a lo que cabría esperar, la de minimización del papel de Francia. Las difíciles relaciones con Argelia y la pretensión de no enemistarse ni con España ni con Marruecos hicieron que Francia no se decantara públicamente.

Por otra parte, Le Monde, periódico dirigido a las elites intelectuales interesadas especialmente en la información internacional, se encontraba en su momento de mayor apogeo. Su tirada y su difusión aumentaban y no se cuestionaba su independencia redaccional después de su posicionamiento particular en la Guerra de Indochina y de Argelia. La cobertura que realizó sobre el tema que nos ocupa respondió a la estrategia deseada por su gobierno: se limitó a ofrecer unos cuantos comunicados oficiales desmintiendo la colaboración con las tropas franquistas en el Sahara en los años cincuenta y silenció totalmente la ayuda francesa a Marruecos en la Marcha Verde (sólo publicó las declaraciones de su presidente reafirmando su neutralidad).

Su análisis resulta útil para observar también la guerra de propaganda generada entre los diversos actores implicados. Es en esta faceta en la que Le Monde responde al periodismo que de él se espera: consulta todas las fuentes y no privilegia a ninguna de ellas en función de la política de su gobierno, sino que las contextualiza y obtiene conclusiones objetivas sobre cómo han podido ocurrir realmente los acontecimientos. Por lo tanto, la abundante información que ofrece nos permite conocer una versión de los dos conflictos que resulta mucho más aproximada a la reconocida después por la historiografía que la de los medios españoles y marroquíes, implicados directamente en la guerra y por tanto más manipulados por su propaganda. 
448 El diario Le Monde y la intervención francesa en el Sahara Occidental

\section{BIBLIOGRAFÍA:}

AGUIRRE, José Fernando: Las Guerras de la postguerra, Barcelona, Argos, 1964 BARBIER, Maurice: Le conflit du Sahara Occidental, París, Éditions L'Harmattan, 1982

BÁRBULO, Tomás: La historia prohibida del Sáhara Español, Barcelona, Destino, 2002

BAUTISTA VILAR, Juan: El Sahara español. Historia de una aventura colonial, Madrid, Sedmay Ediciones, 1977

BOSQUE COMA, Alfredo: Guerra de Ifni, Las Banderas Paracaidistas 1957-1958, Madrid, Almeda, 1998

CASAS DE LA VEGA, Rafael: La última Guerra de África (campaña de Ifni-Sahara) Madrid, Servicio de Publicaciones del EME, 1985

DIEGO AGUIRRE, José Ramón: La última guerra colonial de España, Ifni-Sahara (19571958), Málaga, Algazara, 1993

FERNÁNDEZ-ACEYTUNO, Mariano: Ifni-Sahara. Una Encrucijada en la Historia de España, Dueñas (Palencia), Ed. Simancas, 2001

INSTITUTO DE ESTUDIOS AFRICANOS: La acción de España en Sahara, Madrid, I.D.E.A., 1971

MARQUINA BARRIO, Antonio: España en la política de seguridad occidental 1939-1986, Madrid, Ejército, 1986

PINIÉS, Jaime de: La descolonización del Sahara: un tema sin concluir, Madrid, Espasa Calpe, 1990

PLATÓN, Miguel: Hablan los militares. Testimonios para la Historia ( 1939-1996), Barcelona, Planeta, 2001

RODRÍGUEZ DE VIGURI, Luis: “Despedidas Vergonzosas”, Historia 16, extra n.ำ 9, Madrid, 1979

RUIZ DE CUEVAS, Teodoro: Apuntes para la Historia política de África, Madrid, Dirección General de Relaciones Culturales, 1971, Vol. 1

SALAS LARRAZABAL, Ramón: El Protectorado de España en Marruecos, Madrid, Mapfre, 1992

SANTAMARÍA QUESADA, Ramiro: Ifni-Sahara. La guerra Ignorada, Madrid, Ediciones Dyrsa, 1984

VILLAR, Francisco: El proceso de autodeterminación del Sahara, Valencia, Ed. Fernando Torres, 1982

(Recibido el 24-02-06, aceptado el 17-05-06) 